\title{
Imaging $180^{\circ}$ Polarization Reversal in Ferroelectric Oxides with Electron Backscatter Diffraction
}

\author{
Matthew J. Burch ${ }^{1}$, Chris M. Fancher ${ }^{1}$, Srikanth Patala ${ }^{1}$, and Elizabeth C. Dickey ${ }^{1}$ \\ 1. Center for Dielectrics and Piezoelectrics, North Carolina State University, Department of Materials \\ Science and Engineering, Raleigh, NC, USA
}

A ferroelectric material's permittivity is dependent on the extrinsic contribution of domain wall displacement during the application of an external field, which can involve the motion of both $90^{\circ}$ and $180^{\circ}$ domain walls. Directly quantifying non- $180^{\circ}$ domain structures through diffraction based techniques is generally straightforward; as the diffraction condition will be different from one side of a domain wall to the other, reflecting the rotation of the unit-cell and resulting in an easily quantifiable metric to determine location of domain wall [1]. Identifying $180^{\circ}$ ferroelectric domains is more difficult, as Friedel's law states that the intensity of a diffracting plane and its inverse will be equal. Fortunately, Friedel's law is broken for dynamical scattering in a non-centrosymmetric crystal system, which is necessary for ferroelectricity. This has led to techniques such as convergent beam electron diffraction (CBED) being utilized for the characterization of $180^{\circ}$ domain structures [1].

More recently, electron backscatter diffraction (EBSD) has been utilized to map $90^{\circ}$ domain structures in ferroelectric oxides [2]. However, utilizing EBSD for imaging $180^{\circ}$ domains has not been possible before, due to limitations in simulating dynamical EBSD patterns. Recently, however, De Graef has developed dynamical diffraction simulation software for EBSD, which we have used to map $180^{\circ}$ polarization reversal in ferroelectric oxides [3].

In this work, we map $180^{\circ}$ ferroelectric domains in periodically poled $\mathrm{LiNbO}_{3}$ single crystals Figure 1 demonstrates the difference in experimental patterns across a $180^{\circ}$ domain boundary in $\mathrm{LiNbO}_{3}$. Figure 2 shows the simulated dynamical patterns of $\mathrm{LiNbO}_{3}$ across a $180^{\circ}$ domain boundary, with the same orientation as for the experimental patterns. Figure $3 \mathrm{~A}$ is a secondary-electron SEM micrograph showing the $180^{\circ}$ domain structures (contrast arises from a difference in the height of each domain variant). Along with the SEM image is traditional EBSD inverse pole figure (IPF) map, emphasizing that traditional indexing in not sensitive to polarization reversal. Figure $3 \mathrm{C}$ demonstrates the mapping of $180^{\circ}$ domains using the dynamical diffraction simulations. Figure $3 \mathrm{C}$, compares the simulated pattern from Figure 2a (positive polarization direction) to the patterns in the map from Figure 3A, by directly comparing the intensity differences between Figure $2 \mathrm{a}$ and the experimental patterns. The dark areas in Figure $3 \mathrm{C}$ correspond to the positive polarization direction. The opposite is true for Figure 3D, where the dark areas correspond to the negative polarization direction.

In conclusion, we demonstrate the ability to map $180^{\circ}$ polarization reversal in ferroelectric oxides using EBSD. This new ability may lead to unprecedented characterization of domain structures in polar materials.

References:

[1] Y. H. HU, H. M. Chan, Z. X. Wen, and M. P. Harmer, J. Am. Ceram. Soc., vol. 69, no. 8, pp. 594602, 1986.

[2] Howell, J. A., Vaudin, M. D., \& Cook, R. F. (2014). Journal of Materials Science, 49(5), 2213-2224. 
[3] P. G. Callahan and M. De Graef, Microsc. Microanal., vol. 19, no. 05, pp. 1255-1265, 2013. [4] The authors acknowledge the funding provided by the Center for Dielectric and Piezoelectrics at NCSU. This material is based upon work supported by the National Science Foundation Graduate Research Fellowship for M.J. Burch under Grant No. DGE-0946818. The authors acknowledge the use of the Analytical Instrumentation Facility (AIF) at North Carolina State University. The authors are grateful to professor Marc De Graef for his many contributions to this work.
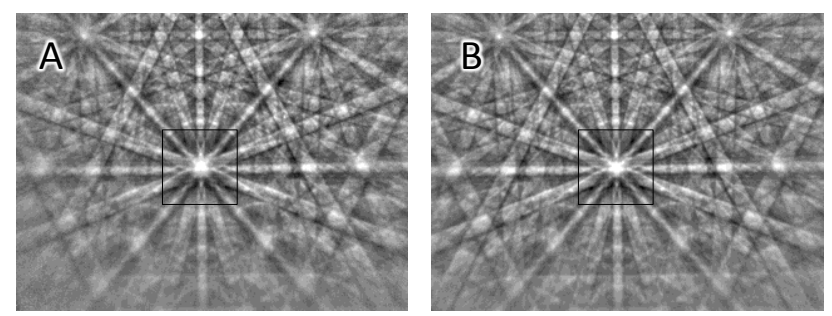

Figure 1. Experimental EBSD patterns from $\mathrm{LiNbO}_{3}$ with (A) and (B) being inverse polarization directions and the inset boxes emphasizing the difference in the patterns.
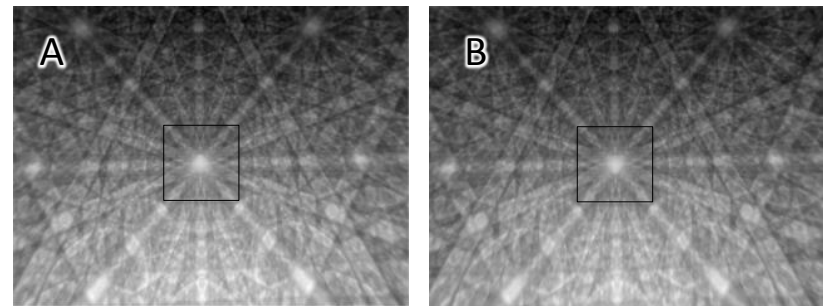

Figure 2. Simulated EBSD patterns with the same orientations as Figure 1.
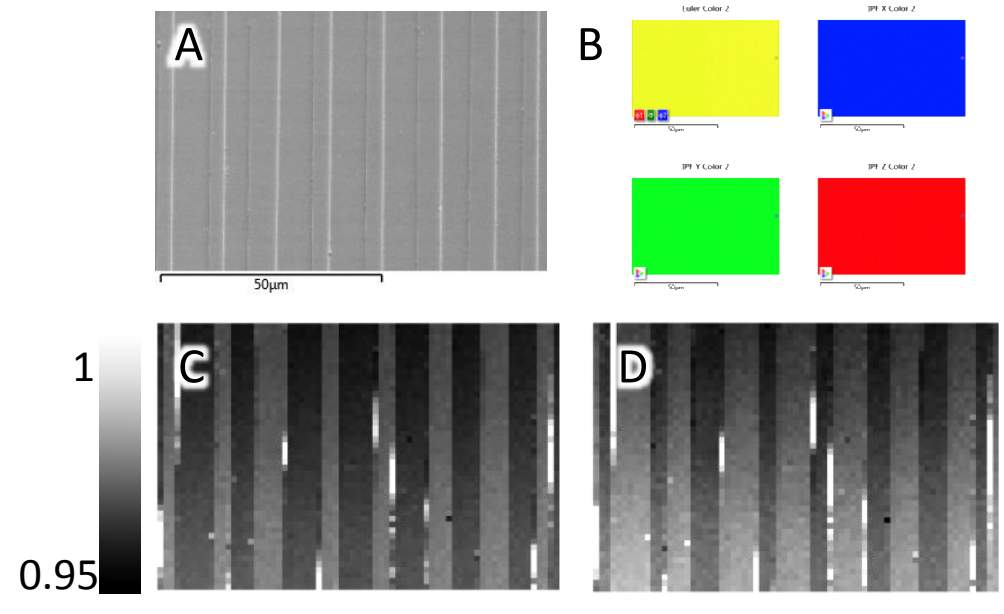

Figure 3. (A) An SEM micrograph of periodically poled $\mathrm{LiNbO}_{3}$ showing the $180^{\circ}$ domain structures. (B) The corresponding IPF maps from EBSD, showing that traditional indexing is not sensitive to polarization inversion. (C) An intensity difference map comparing the experimental patterns from $3 \mathrm{~A}$ to the simulated pattern shown if figure $2 \mathrm{~A}$ demonstrating $180^{\circ}$ domains, with the dark regions have polarization directed out-of-the-page. (D) An intensity difference map comparing the experimental patterns from $3 \mathrm{~A}$ to the simulated pattern shown if figure $2 \mathrm{~B}$ demonstrating $180^{\circ}$ domains, with the dark areas have polarization direction into-the-page. 